\title{
Effect of Hall Current on Mixed Convection Squeezing Three- Dimensional Flow in a Rotating Channel with Thermal Radiation and Heat Sources
}

\author{
Suguna H.G ${ }^{1}$, Bhuvana Vijaya $\mathbf{R}^{2}$ \\ ${ }^{I}$ Assistant Professor, Department of Mathematics, Government Degree College for Boys, Chintamani, \\ Chikkaballapur (Dt), Karnataka, India \\ ${ }^{2}$ Professor of Mathematics College of Engineering, J.N.T.U.A. Anantapuramu, A.P., India
}

*Corresponding Author: Suguna H.G, Assistant Professor, Department of Mathematics, Government Degree College for Boys, Chintamani, Chikkaballapur (Dt), Karnataka, India

\begin{abstract}
We study effect of Hall current on the three-dimensional squeezing flow of an electrically conducting in a rotating channel and its heat and mass transfer characteristics. The governing equations are reduced to set of ordinary differential equations and then numerically solved by employing Runge-KuttaFehlberg fourth-fifth order method. Effect of pertinent parameters on velocity, temperature and concentration fields is examined through the plots. Skin-friction coefficients, Nusselt number and Sherwood number for different variations are studied numerically.
\end{abstract}

Keywords : Hall current, Rotation, Squeezing, Thermo-diffusion, Thermal radiation, Heat sources

\section{INTRODUCTION}

The rotating viscous flow equation yields a layer known as Eckman boundary layer after the Swedish oceanographer Eckman who discovered it. The motion of rotation fluids enclosed with in a body or vice versa, was given by Greenspan [22], discussed these problems relating to the boundary layers and their interaction in rotating flows and gave so many examples relating to such interaction. Several Researchers [Mahendra Mohan [36] Mahendra Mohan and Srivastava [37], Rao et.al. [44] ,Sarojamma and Krishna [47],Krishna et.al. [31], Seth and Ghosh [49], Agarwal and Dhanpal [2], Ghosh [20], El-Mistikawy et.al. [19], Hazim Ali Attia [26], Circar and Mukherjee [16] ,Balasubramanyam [10] and Madhusudhana Reddy [33] ,Singh and Mathew [53] ,Madhavilatha et al[32] ,Sukanya et al[59]] have discuss effect of rotation on convective heat transfer in different configuration under varied conditions.

Rajasekhar et. al. [45] have analysed the effect of Hall current, thermal radiation and thermo-diffusion on convective heat and mass transfer flow of a viscous rotating fluid past a vertical porous plate embedded in a porous medium. Jafarunnisa [28] has discussed the effect of thermal radiation and thermo diffusion on unsteady convective heat and mass transfer flow in the rotating system with heat sources. Alam et. al. [4] have discussed the steady MHD combined heat and mass transfer flow through a porous medium past an infinite vertical plate with viscous dissipation and joule heating effects in a rotating system. Srirangavani [56] has considered the effect of thermo-diffusion on convective heat and mass transfer flow with radiation absorption. Jayasudha et al [29] have analysed the effect of thermo-diffusion on convective heat and mass transfer flow of viscous electrically conducting fluid in a vertical rotating plate in the presence of transverse magnetic field.

The effect of thermo diffusion, thermal radiation on convective heat and mass transfer in a rotating fluid have been investigated by several researchers [1, 5, 12, 28, 29,30, 32, 33, 40, 54, 56, 57, 59, 62].

On the other hand, an unsteady squeezing flow of an electrically conducting fluid occurs in many engineering and industrial applications such as lubrication, food industries, transient loading of mechanical components, power transmission, polymer processing, compression and injection modelling. The squeezing flow of a fluid was first introduces by Stefan [58], Following this work, many researchers have investigated such flow with different aspects. Numerical solution for a fluid 
film squeezed between two parallel plane surfaces have been reported by Hamza and Macdonald [24]. Domairry and Aziz [18] studied the squeezing flow of viscous fluid between parallel disks with suction or blowing analytically. Heat and mass transfer in the unsteady squeezing flow between parallel plates is analyzed by Mustafa et al [39], Hamza [23] discussed the effect of suction and injection on the squeezing flow between parallel plates. It is noted that very little attention has been given to study the three-dimensional flow in a rotating channel. Munawar et al [38] studied the threedimensional flow in a rotating channel of lower stretching sheet in the presence of MHD effects. The mathematical equations are modelled with the help of Navier-Stokes equation and then they are solved numerically. Hayat et al [25] have discussed an unsteady mixed convection three-dimensional squeezing flow of an incompressible Newtonian fluid between two vertical parallel planes. Mahantesh et al[34] have discussed the heat and mass transfer effects on the mixed convective flow of chemically reacting nanofluid past a moving / stationary vertical plate. Mahanthesh et al[34] have studied mixed MHD convection squeezing three-dimensional flow in a rotating channel filled with nanofluid.Sreevani(57a) has discussed the effect of squeezing on MHD convective heat and mass transfer flow of a rotating fluid in a vertical channel 888888888888.Kalpana et al(30a) have studied mixed convection squeezing three-dimensional flow in a rotating channel with thermal radiation and heat sources. A few recent studies [3-14] regarding MHD heat and mass transfer with different physical conditions.

The radiative heat transfer has encountered a variety of applications such as nuclear power plants, gas turbines and propulsion devices for space vehicles, missiles and aircraft. Additionally, heat and mass transfer under the influence of chemical reaction has also attracted considerable attention of many authors due to their wide range of applications.Keeping this in view, conjugate heat and mass transfer flow past a vertical permeable plate with thermal radiation and chemical reaction is investigated by Pal and Talukdar[43]. They found that, the thermal radiation and chemical reaction effect decreases the velocity and concentration profiles.Numerical investigation of heat and mass transfer of an electrically conducting fluid over aa moving surface with first order chemical reaction effect is carried out by Chamkha[15]. Uwanta and Omokhuale[61] have analysed the influence of radiation and chemical reaction effect on heat and mass transfer of a viscoelastic fluid in a fixed plane. The combined effects of radiation and chemical reaction on the MHD free convection flow of an electrically conducting viscous fluid over an inclined plate have been studied by Aly et al [6] and have obtained a closed form solution by employing Laplace transform technique. Das et al [17] have discussed the effect of radiation on unsteady free convective heat and mass transfer flow using Laplace transform method. Several attempts have been made to analyse the effect of thermal radiation and chemical reaction under different physical situations [Seth et al[49], Das[17], Muthucumaraswamy[41], Makinde[35], Gireesha[21], Turkyilmazoglu[60], Sheikholeslami[52], Nanda et al[42,Pal et al[43, Seth et al[50, Seth et al [51], Soundalgekar et al [55]].

In this paper, we study effect of Hall current on the three-dimensional squeezing flow of an electrically conducting in a rotating channel and its heat and mass transfer characteristics. To the best of author's knowledge, such study has not received attention in the engineering sciences literature thus far. The governing equations are reduced to set of ordinary differential equations and then numerically solved by employing Runge-Kutta-Fehlberg fourth-fifth order method. Effect of pertinent parameters on velocity, temperature and concentration fields is examined through the plots. Skinfriction coefficients, Nusselt number and Sherwood number for different variations are studied numerically. The authors have hope that the results obtained in the present study not only provide useful information for applications, it also serves as a complement to the previous studies.

\section{Mathematical Formulation}

Consider an unsteady three-dimensional squeezing flow of an electrically conducting incompressible viscous fluid in a vertical rotating channel. The plane positioned at $y=0$ is stretched with velocity $U_{w o}$ $=a x /(1-\alpha t)$ in $x$-direction and maintained at the constant temperature $\mathrm{T}_{0}$ and concentration Co. The temperature at the other plane is $T_{h}$ and located at a variable distance $h(t)=\sqrt{v_{f}(1-\alpha t)}$. In negative $y$-direction, the fluid is squeezed with a time dependent velocity $V_{h}=d h / d t=-\alpha / 2 \sqrt{v_{f} / \alpha(1-\alpha t)}$. The fluid and the channel are rotated about $y$-axis with angular velocity $\vec{\Omega}=\omega \hat{j} /(1-\alpha t)$. The transverse magnetic field is assumed to be variable kind 
Effect of Hall Current on Mixed Convection Squeezing Three-Dimensional Flow in a Rotating Channel with Thermal Radiation and Heat Sources

$\vec{B}=B_{0} / \sqrt{(1-\alpha t)}$ and it is applied along $y$-axis. The fluid is sucked/injected from the plane located at $y=0$ as shown in figure 1.The strength of the heat source $\mathrm{Q}$ and chemical reaction parameter $\mathrm{kc}$ are assumed to be $Q=\frac{Q_{0}}{(1-\omega t)}, k_{c}^{*}=\frac{k c}{(1-\kappa t)}$ The magnetic Reynolds number is assumed to be small thus induced magnetic field is negligible. In addition, effects viscous dissipation and Joule heating are neglected.

Under those assumptions, the governing equations for the velocity, temperature and Concentration fields in the presence of internal heating source/sink and Hall effects are given by [Hayat et al 2015, Munawar et al 2012].

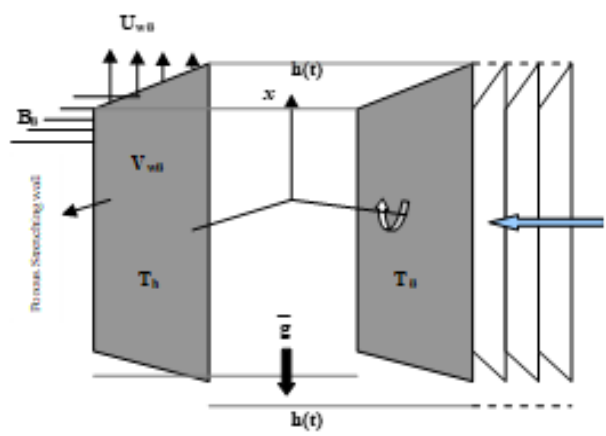

Fig1. Flow configuration and coordinate

$\frac{\partial u}{\partial x}+\frac{\partial V}{\partial y}=0$

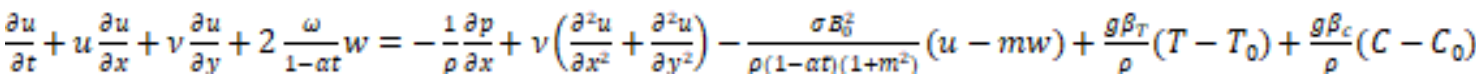

$\frac{\partial u}{\partial t}+u \frac{\partial u}{\partial x}+v \frac{\partial u}{\partial y}=-\frac{1}{\rho} \frac{\partial p}{\partial y}+v\left(\frac{\partial^{2} v}{\partial x^{2}}+\frac{\partial^{2} v}{\partial y^{2}}\right)$.

$\frac{\partial w}{\partial t}+u \frac{\partial w}{\partial x}+v \frac{\partial w}{\partial y}-2 \frac{w}{1-\alpha t} u=v_{v f}\left(\frac{\partial^{2} w}{\partial x^{2}}+\frac{\partial^{2} w}{\partial y^{2}}\right)-\frac{\sigma B_{0}^{2}}{p p[1-\alpha t)\left(1+m^{2}\right)}(m u-w) x$

$\frac{\partial T}{\partial t}+u \frac{\partial T}{\partial x}+v \frac{\partial T}{\partial y}=\frac{k_{f}}{\left\{\rho C_{p}\right)}\left(\frac{\partial^{2} T}{\partial x^{2}}+\frac{\partial^{2} T}{\partial y^{2}}\right)+\frac{Q_{0}}{\left(\rho C_{p}\right)(1-\alpha t)}\left(T-T_{0}\right)-\frac{1}{\left(\rho C_{p}\right)} \frac{\partial\left(q_{k}\right)}{\partial y}$

$\frac{\partial C}{\partial t}+u \frac{\partial C}{\partial x}+v \frac{\partial C}{\partial y}=D_{B}\left(\frac{\partial^{2} C}{\partial x^{2}}+\frac{\partial^{2} C}{\partial y^{2}}\right)+\frac{D_{B} R_{T}}{T_{M}}\left(\frac{\partial^{2} T}{\partial x^{2}}+\frac{\partial^{2} T}{\partial y^{2}}\right)-\frac{k_{C}}{([1-C t)}\left(C-C_{0}\right)$

where $u, v$ and $w$ are velocity components along $x, y$ and $z$ directions respectively, $p$ is pressure. $B_{0}$ is the magnetic field, $\sigma$ is the electrical conductivity, $g$ is the magnitude of acceleration due to gravity, $\alpha$ is characteristic parameter with the dimension of reciprocal of time $t$ and $\alpha t<1$. T is temperature of the fluid ,C is the concentration, $\mathrm{kc}$ is the chemical reaction coefficient, $Q_{0}$ is uniform volumetric heat generation/ absorption; here $Q_{0}<0$ and $Q_{0}>0$ are respectively corresponds to internal heat absorption and generation,Dm is the molecular diffusivity, $\mathrm{Tm}$ is the mean fluid temperature, $\mathrm{K}_{\mathrm{T}}$ is the thermal diffusion ratio, $\rho_{n f}$ is effective density of the nanofluid, $v_{n f}=\mu_{n f} / \rho_{n f}$ is effective kinematic viscosity of the nanofluid, $k_{f}$ and $\left(\rho C_{p}\right)$ are thermal conductivity and heat capacity of the fluid respectively. $\rho_{f}$ is the density of base fluid, $\mu_{f}$ is the dynamic viscosity of the base fluid, $\left(\rho C_{p}\right)_{f}$ is the heat capacity of the base fluid, $k_{f}$ is the thermal conductivity of the base fluid The approximate boundary conditions for the present problem are;

$u(x, y, t)=U_{w 0}, v(x, y, t)=V_{w 0}$,

$w(x, y, t)=0, T(x, y, t)=T_{0}, C(x, y, t)=C_{0}, \quad$ at $y=0$

$u(x, y, t)=0, v(x, y, t)=V_{h}$,

$w(x, y, t)=0, T(x, y, t)=T_{h}, C(x, y, t)=C_{h} \quad$ at $y=h(t)$

where $T_{h}=T_{0}+T_{0} /(1-\alpha t), C_{h}=C_{0}+C_{0} /(1-\alpha t), V_{w 0}=-V_{0} /(1-\alpha t)$. Here $V_{0}$ is constant, $V_{w 0}<0$ corresponds injection whereas $V_{w 0}>0$ corresponds wall suction. 
To reduce the governing equations into a set of similarity equations, introduce the following similarity transformations [Munawar et al 2012].

$\psi=\sqrt{\frac{\alpha v_{f}}{1-\alpha t}} x f(\eta), \eta=\frac{y}{h(t)}, T=T_{0}+\frac{T_{0}}{1-\alpha t} \theta(\eta), C=C_{0}+\frac{C_{0}}{1-\alpha t} C(\eta)$,

$u=U_{w 0} f_{\eta} \eta, v=-\sqrt{\frac{\alpha v_{f}}{1-\alpha t}} f(\eta), w=U_{w 0} g(\eta)$,

where a suffix $\eta$ denote the differentiation with respect to $\eta$ and $v_{f}$ is the kinematic viscosity of the fluid. Using the above transformations (2.9), the equation (2.1) is automatically satisfied, while the equation $(2.2)-(2.5)$ are respectively reduces to the following nonlinear ordinary differential equations;

$$
\begin{aligned}
& f_{\eta \eta \eta}=\left[f f_{\eta \eta}-f_{\eta}^{2}-\beta\left(f_{\eta} \frac{\eta}{2} f_{\eta \eta}\right)-2 R g-\frac{M^{2}}{1+m^{2}}\left(f_{\eta}+m g\right)+G(\theta+N C)\right] \\
& =\frac{(1-\alpha t)^{2}}{\rho a^{2} x} \frac{\partial p}{\partial x^{\eta}} \\
& f_{\eta \eta}-\left[-f f_{\eta}+\frac{\beta}{2}\left(f+\eta f_{\eta}\right)\right]=-\frac{1-\alpha t}{\rho v_{f} a} p_{\eta \eta} \\
& g_{\eta \eta}+\left[f g_{\eta}-f_{\eta} g-\beta\left(g \frac{\eta}{2} g_{\eta}\right)+2 R f_{\eta}\right]-\frac{M^{2}}{1+m^{2}}\left(m f_{\eta}-m g\right)=0, \\
& \left(1+\frac{4 N r}{3}\right) \theta_{\eta \eta}+\operatorname{Pr}\left[\left\{\beta\left(\theta+\frac{\eta}{2} \theta_{\eta}\right)+f \theta_{\eta}\right\}-Q \theta\right]=0 . \\
& C_{\eta \eta}+S c\left[\left\{\beta\left(C+\frac{\eta}{2} C_{\eta}\right)+f C_{\eta}\right\}+S r \theta_{\eta \eta}-\gamma C\right]=0
\end{aligned}
$$

Reduced boundary conditions are;

$f_{\eta}=1, \quad f=S, \quad g=0, \quad \theta=0, \mathrm{C}=0 \quad$ at $\quad \eta=0$

$f_{\eta}=0, \quad f=\frac{\beta}{2}, g=0, \quad \theta=1 \quad, \mathrm{C}=1 \quad$ at $\quad \eta=1$

where

$\beta=\alpha / \alpha$ is the squeezing parameter, $R=\omega / \alpha$ is rotation parameter, $M^{2}=\sigma B_{0}^{2} / \alpha \rho_{f}$ is magnetic parameter, $G r=G / R e^{2}$ is mixed convection parameter, $G=g \beta_{T} T_{0} x^{3} / v_{f}^{2}(1-\alpha t)$ is Grashaf number, $R e=x U_{w 0} / v_{f}$ is Reynolds number, $N=\beta_{c} C_{0} / \beta_{T} T_{0}$ is the buoyancy ratio, $\operatorname{Pr}=\left(\mu c_{p}\right)_{\mathrm{f}} / k_{f}$ is the Prandtl number, $S c=\frac{v_{f}}{D_{m}} \quad$ is the Schmidt number, $S r=\frac{D_{m} K_{T} T_{0}}{a T_{m} C_{\mathrm{n}}}$ is the Soret parameter,$\gamma=\frac{k \epsilon}{a}$ is the chemical reaction parameter, $Q=Q_{0} / \alpha\left(\rho C_{p}\right)_{f}$ is heat source / sink parameter $N r=\frac{4 \sigma^{*} T_{o}^{a}}{\beta_{R^{k}} k_{f}}$ is the radiation parameter and $S=V_{w 0} / \alpha h$ is suction / injection parameter.

It is important to mention that, $\beta=0$ represents plates are stationary, $\beta>0$ corresponds to the plate which is located at $y=h(t)$ moves towards the plate which is located at $y=0$ and $\beta<0$ corresponds to the plate at $y=y(t)$ moves apart with respect to the plate at $y=0$.

Now in order to reduce the number of independent variables by cross differentiation; the set of equations (2.10)-(2.13) takes the following form;

$$
\begin{aligned}
& f_{\eta \eta \eta}-\left[\frac{\beta}{2}\left(3 f_{\eta \eta}+\eta f_{\eta \eta \eta}\right) f_{\eta \eta} f_{\eta \eta}-f f_{\eta \eta \eta}+2 R g_{\eta}\right] \\
& \left.-\frac{M^{\mathrm{I}}}{1+m^{\mathrm{n}}}\left(f_{\eta \eta}+m g_{\eta}\right)+m g\right)+G\left(\theta_{\eta}+N C_{\eta \eta}\right)=0 \\
& g_{\eta \eta}+\left[f g_{\eta}-f_{\eta} g-\beta\left(g+\frac{\eta}{2} g_{\eta}\right)+2 R f_{\eta}\right]-\frac{M^{\mathrm{x}}}{1+m^{\mathrm{a}}}\left(m f_{\eta}-g\right)=0 \\
& \left(1+\frac{4 N r}{3}\right) \theta_{\eta v}-\operatorname{Pr}\left(\left\{\beta\left(\theta+\frac{\eta}{2} \theta_{\eta}\right)+f \theta_{n}\right\}-Q \theta\right)=0 \\
& c_{\eta \eta}-S c\left(\left\{\beta\left(c+\frac{\eta}{2} C_{\eta}\right)+f \theta_{\eta}\right\}-\gamma C+\operatorname{Sr} \theta^{\mu}\right)=0
\end{aligned}
$$


Effect of Hall Current on Mixed Convection Squeezing Three-Dimensional Flow in a Rotating Channel with Thermal Radiation and Heat Sources

For engineering and industrial point of view, one has usually less interest in velocity and temperature profiles nature than in the value of the skin-friction and rate of heat transfer. Therefore expression for the local skin-friction coefficient and the local Nusselt number at both the walls are defined as;

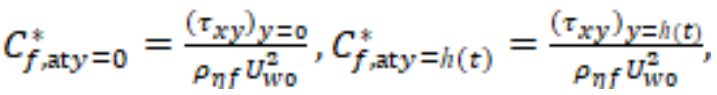

$$
\begin{aligned}
& N u_{\mathrm{aty} y=0}^{*}=\sqrt{\frac{v_{f}}{a}} \frac{\left(q_{x y}\right)_{y=0}}{k_{f} T_{0}}, N u_{\mathrm{at} y=h(t)}^{*}=\sqrt{\frac{v_{f}}{a}} \frac{\left(q_{x y}\right)_{y=h(t)}}{k_{f} T_{0}}, \\
& S h_{\text {aty }=0}^{*}=\sqrt{\frac{v_{f}}{a}} \frac{\left(m_{x y}\right)_{y=0}}{D_{m} c_{0}}, S h_{\text {aty }=h(t)}^{*}=\sqrt{\frac{v_{f}}{a}} \frac{\left(m_{x y}\right)_{y=h(t)}}{D_{m} C_{0}},
\end{aligned}
$$

where $\tau_{x y}$ is the shear stress, $q_{x y}$ is the heat flux, and $\mathrm{m}_{\mathrm{xy}}$ is the mass flux which are given by

$\tau_{x t}=\mu_{\eta f f}\left(\frac{\partial u}{\partial x}+\frac{\partial u}{\partial y}\right), q_{x t}=-k_{\eta f}\left(\frac{\partial T}{\partial x}+\frac{\partial T}{\partial y}\right)$ and $m_{x t}=-D_{m}\left(\frac{\partial C}{\partial x}+\frac{\partial C}{\partial y}\right)$

In view of equation (2.23) and similarity transformations (2.9); equations (2.20)-(2.22) will takes the following form;

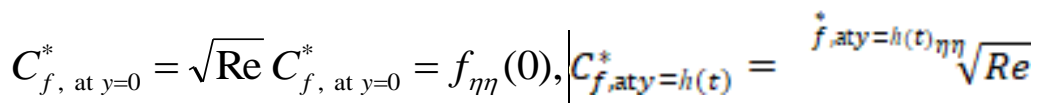

$$
\begin{aligned}
& N u_{\text {aty } y=0}=(1-\alpha t)^{1,5} N u_{\text {at } y=0}^{*}=-\theta_{\eta}(0), N u_{\text {aty }=h(t)}=(1-\alpha t)^{1,5} N u_{\text {aty } y=h(t)}^{*}=-\theta_{\eta}(1) \\
& S h_{\mathrm{aty}=0}=(1-\alpha t)^{1,5} S h_{\mathrm{aty}=0}^{*}=-C_{\eta}(0), S h_{\mathrm{aty}=h(t)}=(1-\alpha t)^{1,5} S h_{\mathrm{aty} y}^{*}=h(t)=-C_{\eta}(1)(2.24)
\end{aligned}
$$

\section{NUMERICAL METHOD AND VALIDATION}

A set of non-similar equations (2.16)-(2.19) are nonlinear in nature and possess no analytical solution, thus, a numerical treatment would be more appropriate. These set of ordinary differential equations together with the boundary conditions (2.15) are numerically solved by employing fourth-fifth order Runge-Kutta-Fehlberg scheme with the help of Maple. This algorithm in Maple is proven to be precise and accurate and which has been successfully used to solve a wide range of nonlinear problem in transport phenomena especially for flow and heat transfer problems. In this study, we set the relative error tolerance to $10^{-6}$. Comparison results are recorded in table 1 and are found to be in excellent agreement. The effects of development of the squeezing three-dimensional flow and heat transfer in a rotating channel for different values of squeezing parameter, rotation parameter, Hall parameter magnetic parameter, suction/injection parameter, mixed convection parameter, radiation

\begin{tabular}{|c|c|c|c|c|c|c|c|c|c|}
\hline \multirow{2}{*}{\multicolumn{2}{|c|}{ Parameter }} & \multicolumn{4}{|c|}{ Kalpana et al [30a] } & \multicolumn{4}{|c|}{ Present results } \\
\hline & & $\tau \mathrm{x}(1)$ & $\tau z(1)$ & $\mathrm{Nu}(1)$ & $\operatorname{Sh}(1)$ & $\tau \mathrm{x}(\mathbf{1})$ & $\tau \mathbf{Z}(\mathbf{1})$ & $\mathrm{Nu}(1)$ & $\operatorname{Sh}(1)$ \\
\hline \multirow[t]{4}{*}{$\mathrm{R}$} & 0.5 & 4.34299 & 0.277668 & -0.999983 & -1.31302 & 4.34293 & 0.277666 & -0.999980 & -1.313002 \\
\hline & 1.0 & 4.30684 & 0.833542 & -0.999988 & -1.31305 & 4.30686 & 0.833541 & -0.999986 & -1.31303 \\
\hline & 1.5 & 4.23431 & 1.391062 & -0.999993 & -1.31311 & $\mathbf{4 . 2 3 4 3 4}$ & 1.391063 & -0.999991 & -1.31309 \\
\hline & 2.0 & 4.12494 & 1.951422 & -0.999999 & -1.31322 & 4.12496 & 1.951426 & -0.999998 & -1.31324 \\
\hline \multirow[t]{4}{*}{$\mathrm{Sr}$} & 0.5 & 4.34299 & 0.277668 & -0.999983 & -1.31301 & 4.34301 & 0.277666 & -0.999987 & -1.31303 \\
\hline & 1.0 & 4.34292 & 0.277664 & -0.999990 & -1.31289 & 4.34290 & 0.277660 & -0.999988 & -1.31286 \\
\hline & 1.5 & 4.34287 & 0.277660 & -0.999994 & -1.312788 & 4.34284 & 0.277656 & -0.999993 & -1.312783 \\
\hline & 2.0 & 4.34322 & 0.277658 & -0.999999 & -1.312335 & 4.34323 & 0.277653 & -0.999997 & -1.312338 \\
\hline \multirow[t]{4}{*}{$\mathrm{Nr}$} & 0.5 & 4.34299 & 0.277668 & -0.99996 & -1.31302 & $\mathbf{4 . 3 4 2 9 7}$ & 0.277665 & -0.99998 & -1.31301 \\
\hline & 1.5 & 4.34302 & 0.277667 & -1.0004 & -1.31368 & 4.34300 & 0.277663 & -1.00044 & -1.31366 \\
\hline & 3.5 & 4.34305 & 0.277665 & -1.00088 & -1.31431 & 4.34304 & 0.277662 & -1.00083 & -1.31430 \\
\hline & 5.0 & 4.34313 & 0.277662 & -1.00219 & -1.31595 & 4.34316 & 0.277659 & -1.00212 & -1.31594 \\
\hline \multirow[t]{4}{*}{$\beta$} & 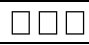 & 4.34299 & 0.277668 & -0.999983 & -1.31302 & 4.34296 & 0.277666 & \begin{tabular}{|l|l|}
-0.99998 \\
\end{tabular} & -1.31300 \\
\hline & 0.4 & 3.67161 & 0.225586 & -0.999977 & -1.36765 & 3.67159 & 0.225580 & -0.999973 & -1.36765 \\
\hline & 0.6 & 3.03904 & 0.177955 & -0.999972 & -1.42139 & 3.03902 & 0.177956 & -0.999970 & -1.42136 \\
\hline & 0.8 & 2.41319 & 0.132139 & -0.999968 & -1.47682 & 2.41313 & 0.132135 & -0.999965 & $\begin{array}{l}-1.47679 \\
\end{array}$ \\
\hline
\end{tabular}
parameter, Prandtl number and heat source/sink parameter. In the following section, the results are discussed in detail with the aid of plotted graphs and tables.

Table1. In the absence of Hall effects $(m=0)$ the results are in good agreement with Kalpana et al [30a] 
We make an investigation of the three-dimensional squeezing convective flow, heat and mass transfer flow of an electrically conducting fluid in a rotating channel in the presence of internal heat generating heat source/sink. In our numerical simulation the default values of telhe parameters are considered as: $\mathrm{M}^{2}=0.5, \mathrm{Q}=0.5 . \mathrm{R}=0.5, \beta=0.5, \mathrm{Pr}=0.71, \mathrm{Sc}=1.3, \mathrm{Sr}=05, \gamma=0.5$.In order to analyse the effects of various pertinent parameters on velocity, temperature and concentration profiles, several graphs are plotted.

Fig.2a-2d represent the effect of magnetic field on $\mathrm{f}^{\prime}, \mathrm{g}, \theta$ and $\mathrm{C}$. It can be found from the profiles that the velocity component $g$ reduces with increase in $m$ in the entire flow region(fig. $3 \mathrm{~b}$ ), while the axial velocity $\mathrm{f}^{\prime}$ 'reduces in the right half $(0,0.5)$ and enhances in the left half $(0.5,1.0)$ of the channel(fig.2a). The thickness of the thermal and solutal boundary layers enhance with increase in $m$ which results in a rise in the temperature and concentration in the flow region(figs.2c\&2d).

The influence of rotation parameter(R) on $\mathrm{f}^{\prime}, \mathrm{g}, \theta$ and $\mathrm{C}$ can be observed from the figs.2a-2d. The transverse velocity $g$ reduces with increase in $\mathrm{R}$. The temperature and concentration distributions experience an enhancement in the entire flow region with increase in rotation parameter (R). This is due to the fact thickness of the thermal and solutal boundary layers increase with $\mathrm{R}$.

Fgs.4a-4d show the influence of thermal radiation on $\mathrm{f}^{\prime}, \mathrm{g}, \theta$ and $\mathrm{C}$. It can be seen from the profiles(figs.6a-6d) that there is a significant depreciation in the magnitude of the velocity component $\mathrm{f}$ 'and $\mathrm{g}$ in the presence of thermal radiation throughout the flow region. The radiation parameter is found to reduce the hydrodynamic boundary layers along $\mathrm{x}$ and $\mathrm{y}$-directions. the presence of the thermal radiation is very significant on the variation of temperature. It is seen that the temperature increases rapidly in the presence of thermal radiation parameter throughout the flow region. This may be attributed to the fact that as the Roseland radiative absorption parameter $\mathrm{R} *$ diminishes the corresponding heat flux diverges and thus raising the rate of radiative heat transfer to the fluid causing a rise in the temperature of the fluid. The thickness of the thermal boundary layer also increases with increase in $\mathrm{Nr}$ (fig.4c). The effect of mass concentration can be seen from fig.4d. We find a depreciation in the concentration with a rise in $\mathrm{Nr}$, owing to a fall in the thickness of the solutal boundary layer.

Figs.5a-5d are plotted to illustrate the effect of heat source parameter (Q) on the flow variables.It can be seen from the profiles that the axial and transverse velocity components reduce in the flow region with increase in the strength of the heat generating source and enhances in the case of heat absorbing source case.This may be attributed to the fact that in the presence of heat generating source,energy is absorbed in the flow region while in the case of heat absorbing heat source,energy is generating in the flow region.(figs.5a-5b). From figs.5c \&5d we find that in the presence of heat generating source,energy is absorbed in the flow region,which results in a reduction in the temperature while in the presence of heat absorbing source,energy is generated which leads to an enhancement in the temperature.The effect of heat source parameter $(\mathrm{Q})$ on the concentration is to reduce $\mathrm{C}$ with $\mathrm{Q}>0$ and enhance with $\mathrm{Q}<0$ in the entire flow region(fig.5d).

The effect of chemical reaction on $\mathrm{f}^{\prime}, \mathrm{g}, \theta$ and $\mathrm{C}$ can be seen from figs.6a-6d.It can be see from the fig.6a the axial velocity and transverse velocity enhances in the degenerating case and reduces in the generating case in the left half while in the right half of the channel ,the axial velocity reduces, transverse velocity enhances for $\gamma>0$ and for $\gamma<0$, the axial velocity enhances ,the transverse velocity reduces in the flow region(figs. $6 \mathrm{a} \& 6 \mathrm{~b}$ ). The temperature and concentration reduce in the degenerating chemical reaction case and enhance in the generating chemical reaction case(figs.6c $\& 6 \mathrm{~d}$ ).

The effect of thermo-diffusion on $\mathrm{f}^{\prime}, \mathrm{g}, \theta$ and $\mathrm{C}$ is depicted in figs.7a-7d.It can be seen from the profiles that higher the thermo-diffusion smaller all the axial, transverse velocity components(figs.7a$7 b)$.From figs.7c\&7d we find that a rise in Soret parameter(Sr) results in an increment in the thermal and solutal boundary layers thickness which leads to a rise in temperature and concentration in the entire flow region.

Figs.8a-8d present the typical profiles namely, $\mathrm{f}^{\prime}, \mathrm{g}, \theta$ and $\mathrm{C}$ respectively for different values of the squeezing parameter $(\beta)$.From figs. $8 \mathrm{a} \& 8 \mathrm{~b}$ show that the magnitude of the axial velocity ( $\left.f^{\prime}\right)$ is an increasing function and the transverse velocity $\mathrm{g}$ is a decreasing function of squeezing parameter. This implies that squeezing effect on flow field is accumulated by it An increase in $\beta$ results in a reduction in the temperature and concentration (figs. $8 \mathrm{c} \& 8 \mathrm{~d}$ ). 

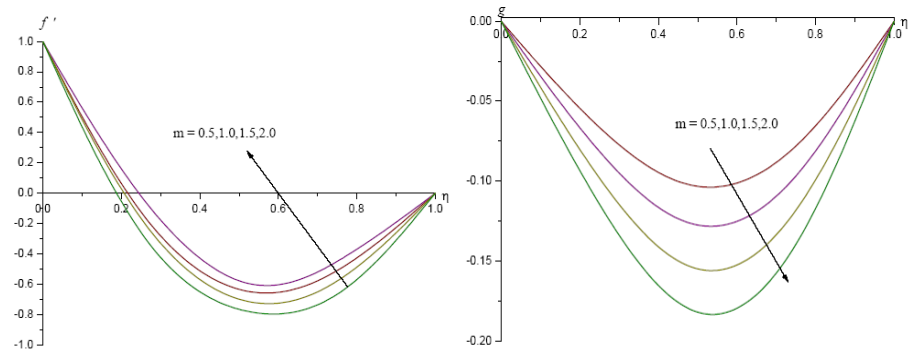

Fig. $2 \mathrm{a}$ effect of $m$ on $\mathrm{f}^{\prime}(\eta)$ profiles $\mathrm{R}=0.5, \mathrm{Q}=0.5, \mathrm{SI}=0.5, \gamma=0.5, \beta=0.5, \mathrm{NI}=0.5$



Fig. $2 \mathrm{~d}$ Effect of $\mathrm{m}$ on $\mathrm{f}^{\prime}(\eta)$ profiles $\mathrm{R}=0.5, \mathrm{Q}=0.5, \mathrm{Sr}=0.5, \gamma=0.5, \beta=0.5, \mathrm{Nr}=0.5$
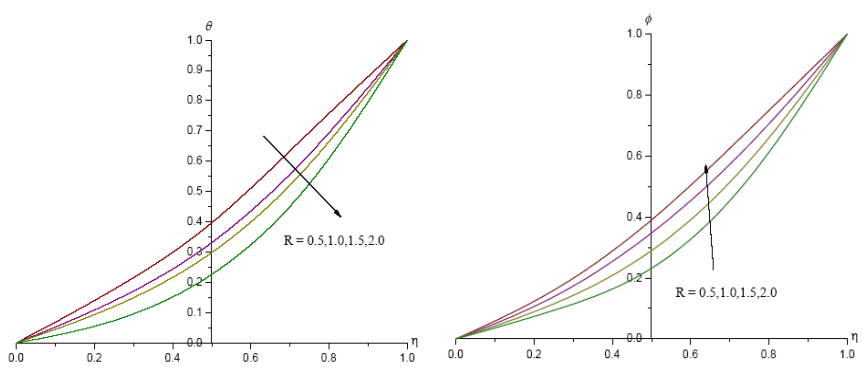
Fig. $3 \mathrm{c}$ Effect of $\mathrm{R}$ on $\theta(\eta)$ profiles
$\mathrm{m}=0.5, \mathrm{Q}=0.5, \mathrm{Sr}=0.5, \gamma=0.5, \beta=0.5, \mathrm{Nr}=0.5$

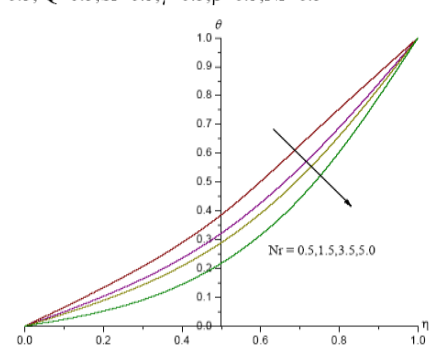

Fig.4c Effect of $\mathrm{Nr}$ on $\mathrm{f}^{\prime}(\eta)$ profiles $\mathrm{R}=0.5, \mathrm{Q}=0.5, \gamma=0.5, \beta=0.5, \mathrm{~m}=0.5, \mathrm{Sr}=0.5$

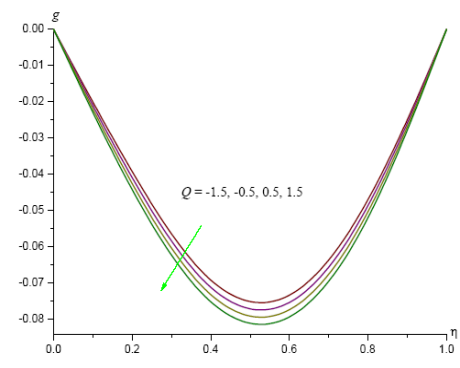

Fig.5b Effect of $Q$ on $g(\eta)$ profiles $\mathrm{R}=0.5, \mathrm{Sr}=0.5, \gamma=0.5, \beta=0.5, \mathrm{Nr}=0.5, \mathrm{~m}=0.5$
Fig. $2 b$ Effect of $m$ on $g(\eta)$ profiles $\mathrm{R}=0.5, \mathrm{Q}=0.5, \mathrm{Sr}=0.5, \gamma=0.5, \beta=0.5, \mathrm{Nr}=0.5$

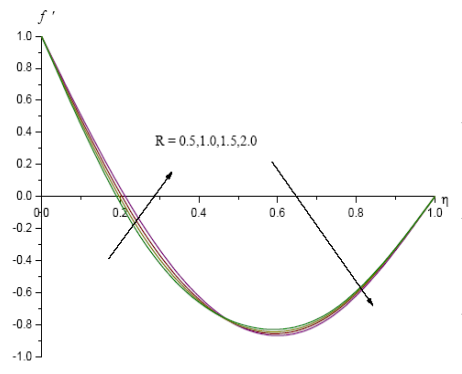

Fig. 3a effect of $R$ on $f^{\prime}(\eta)$ profiles $\mathrm{m}=0.5, \mathrm{Q}=0.5, \mathrm{Sr}=0.5, \gamma=0.5, \beta=0.5, \mathrm{Nr}=0.5$

Fig.3d Effect of R on $\phi(\eta)$ profiles $\mathrm{m}=0.5, \mathrm{Q}=0.5, \mathrm{Sr}=0.5, \gamma=0.5, \beta=0.5, \mathrm{Nr}=0.5$



Fig. $4 \mathrm{~d}$ Effect of $\mathrm{Nr}$ on $\mathrm{f}^{\prime}(\eta)$ profiles $\mathrm{R}=0.5, \mathrm{Q}=0.5, \gamma=0.5, \beta=0.5, \mathrm{~m}=0.5, \mathrm{Sr}=0$ s

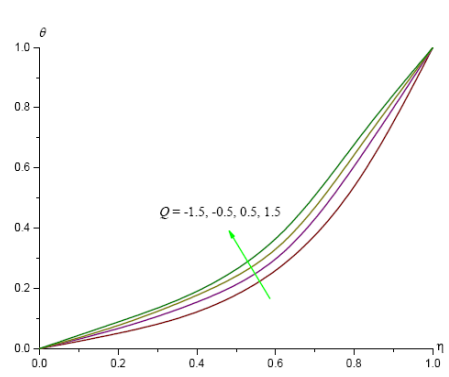

Fig. $5 \mathrm{c}$ Effect of $\mathrm{Q}$ on temperature $\theta(\eta)$ profiles $\mathrm{R}=0.5, \mathrm{Sr}=0.5, \gamma=0.5, \beta=0.5, \mathrm{Nr}=0.5, \mathrm{~m}=0.5$

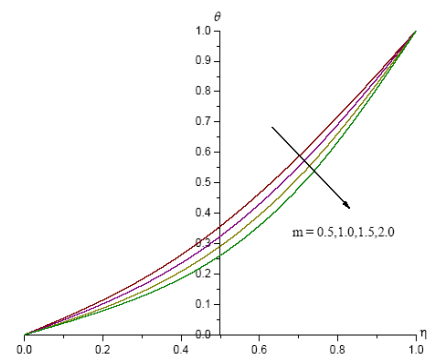

Fig.2c Effect of $m$ on $\mathrm{f}^{\prime}(\eta)$ profiles $\mathrm{R}=0.5, \mathrm{Q}=0.5, \mathrm{Sr}=0.5, \gamma=0.5, \beta=0.5, \mathrm{Nr}=0.5$

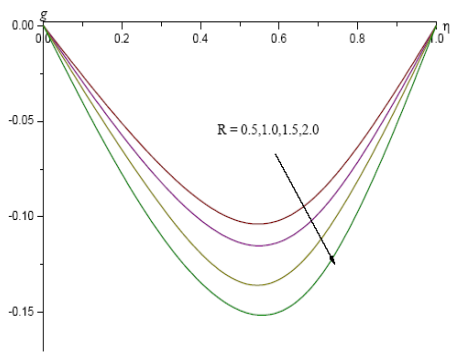

Fig. $3 \mathrm{~b}$ effect of $\mathrm{R}$ on $\mathrm{g}(\eta)$ profiles $\mathrm{m}=0.5, \mathrm{Q}=0.5, \mathrm{Sr}=0.5, \gamma=0.5, \beta=0.5, \mathrm{Nr}=0.5$

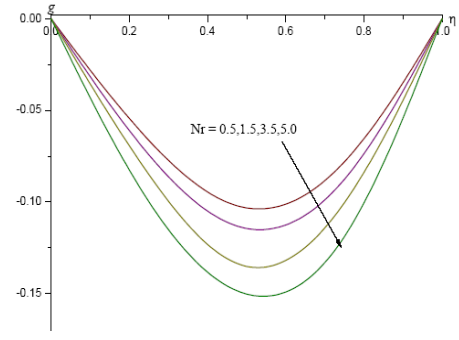

Fig. $4 \mathrm{~b}$ Effect of $\mathrm{Nr}$ on $\mathrm{f}^{\prime}(\eta)$ profiles $\mathrm{R}=0.5, \mathrm{Q}=0.5, \gamma=0.5, \beta=0.5, \mathrm{~m}=0.5, \mathrm{Sr}=0.5$

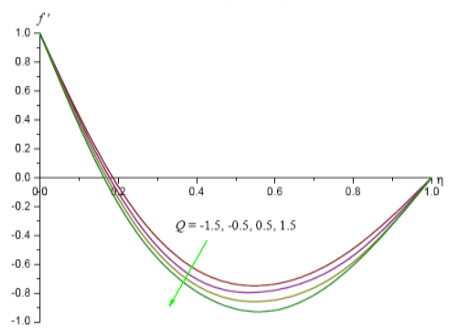

Fig. 5a Effect of $Q$ on $\mathrm{f}^{\prime}(\eta)$ profiles $\mathrm{R}=0.5, \mathrm{Sr}=0.5, \gamma=0.5, \beta=0.5, \mathrm{Nr}=0.5, \mathrm{~m}=0$.

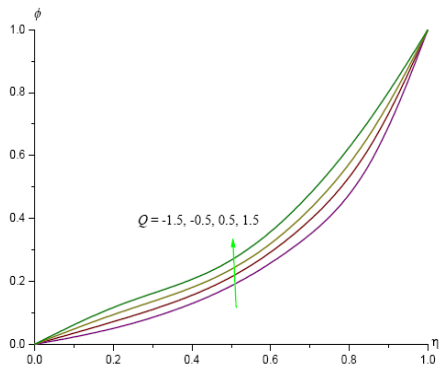

Fig. $5 \mathrm{~d}$ Effect of $\mathrm{Q}$ on Concentration $\phi(\eta)$ profiles $\mathrm{R}=0.5, \mathrm{Sr}=0.5, \gamma=0.5, \beta=0.5, \mathrm{Nr}=0.5, \mathrm{~m}=0.5$ 

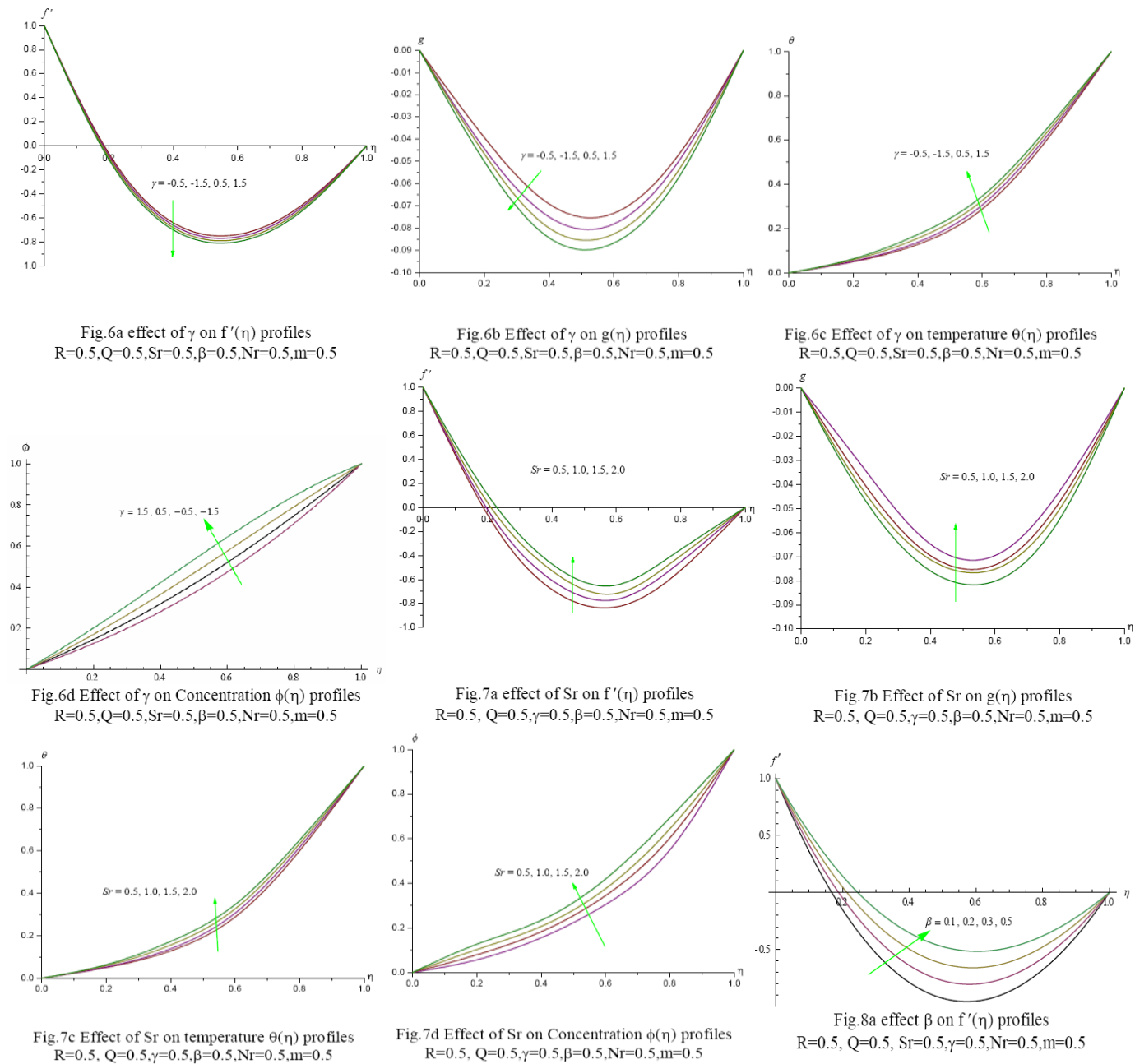
$\mathrm{R}=0.5, \mathrm{Q}=0.5, \gamma=0.5, \beta=0.5 \mathrm{Nr}=0.5 \mathrm{~m}=0.5$

Fig. $7 \mathrm{~b}$ Effect of $\mathrm{Sr}$ on $\mathrm{g}(\eta)$ profiles $\mathrm{R}=0.5, \mathrm{Q}=0.5, \gamma=0.5, \beta=0.5, \mathrm{Nr}=0.5, \mathrm{~m}=0.5$

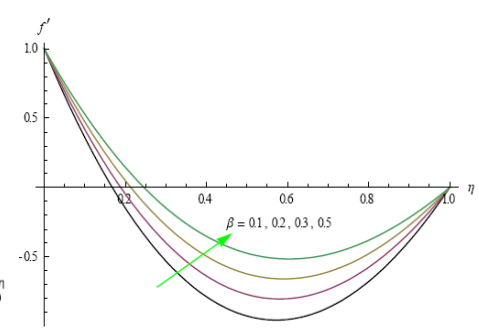

Fig.8a effect $\beta$ on $\mathrm{f}^{\prime}(\eta)$ profiles $\mathrm{R}=0.5, \mathrm{Q}=0.5, \mathrm{Sr}=0.5, \gamma=0.5, \mathrm{Nr}=0.5, \mathrm{~m}=0.5$
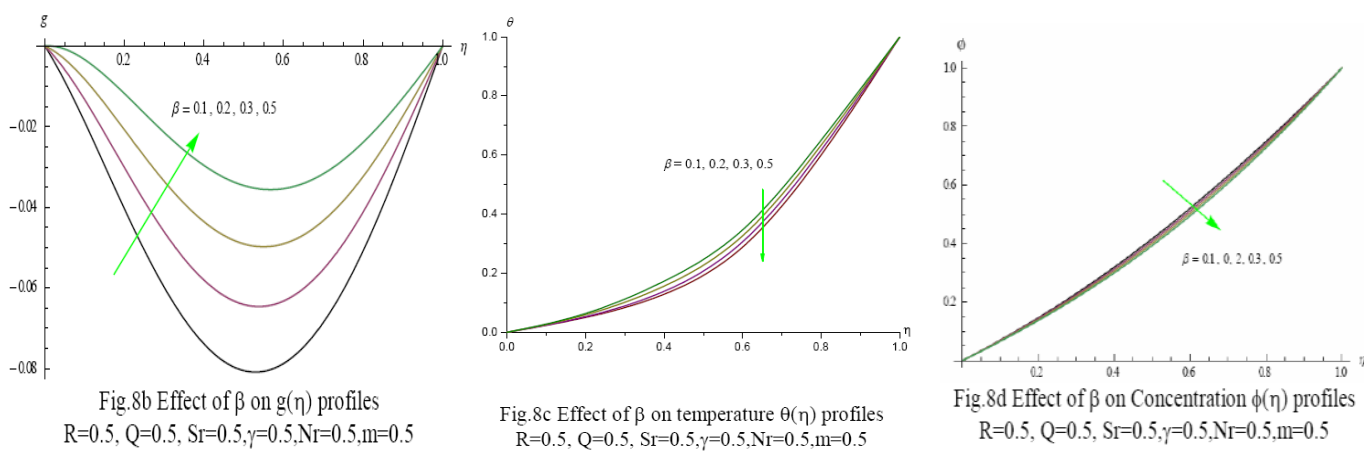

Fig. $8 \mathrm{c}$ Effect of $\beta$ on temperature $\theta(\eta)$ profiles
$\mathrm{R}=0.5, \mathrm{Q}=0.5, \mathrm{Sr}=0.5, \gamma=0.5, \mathrm{Nr}=0.5, \mathrm{~m}=0.5$ $\mathrm{R}=0.5, \mathrm{Q}=0.5, \mathrm{Sr}=0.5, \gamma=0.5, \mathrm{Nr}=0.5, \mathrm{~m}=0.5$

The Skin friction components $\tau x, \tau y$, Nusselt number and Sherwood number on the walls $((\eta=0,1)$ are exhibited in tables. 2 for different parametric variations. An increase in Hall parameter(m)/rotation parameter $(\mathrm{R})$ reduce $\tau \mathrm{X}$ and enhances $\tau \mathrm{Z}$ on both walls. Higher the radiative heat flux larger the skin friction components on the right wall while on the left wall, $\tau \mathrm{x}$ reduces, $\tau \mathrm{z}$ enhances on the left wall. Lesser the molecular diffusivity larger the skin friction components $\tau \mathrm{x}, \tau \mathrm{Z}$ on both the walls while larger $\tau \mathrm{x}$ and smaller $\tau \mathrm{z}$ on $\eta=1$.The variation of skin friction components with heat source parameter $(\mathrm{Q})$ shows that $\tau \mathrm{x}, \tau \mathrm{z}$ enhances with increase in the strength of the heat generating source and depreciates with that of heat absorption source at the left wall while on the right wall $(\eta=1), \tau \mathrm{x}, \tau \mathrm{z}$ increase with $\mathrm{Q}>0$ and $\mathrm{Q}<0$.With respect to chemical reaction parameter $(\gamma)$,we find that $\tau \mathrm{x}, \tau \mathrm{Z}$ reduce at the left wall $(\eta=0)$ in both the degenerating and generating chemical reaction cases, while on the right wall $(\eta=1), \tau \mathrm{x}$ reduces and $\tau \mathrm{z}$ reduces in the degenerating case, in generating case, $\tau \mathrm{x}, \tau \mathrm{z}$ reduces. Higher the thermo-diffusion effect larger $\tau \mathrm{x}$ and smaller $\tau \mathrm{z}$ at the left wall while on the right wall, $\tau \mathrm{x}, \tau \mathrm{z}$ enhances with increase in Sr.An increase in the squeezing parameter $(\beta)$ smaller $\tau \mathrm{x}$ and $\tau \mathrm{z}$ at both the walls. 
Effect of Hall Current on Mixed Convection Squeezing Three-Dimensional Flow in a Rotating Channel with Thermal Radiation and Heat Sources

The rate of heat transfer $(\mathrm{Nu})$ and mass transfer $(\mathrm{Sh})$ at $\eta=0,1$ are shown in table.2. The rate of heat transfer reduces at the left wall and enhances at the right wall with increase in $\mathrm{Nr}$. An increase in Hall parameter $(\mathrm{m})$ /rotation parameter( $\mathrm{R})$ reduces $\mathrm{N} u$ at both the walls. Higher the themo-diffusion effects/squeezing effects/smaller the thermo-diffusivity smaller $\mathrm{Nu}$ at the right wall $(\eta=0)$ and larger at the right wall $(\eta=1)$. When the molecular buoyancy force dominates over the thermal buoyancy force $\mathrm{Nu}$ reduces at the left wall and increases at the right wall irrespective of the directions of the buoyancy forces. An increase in $\mathrm{Q}>0 / \mathrm{Q}<0$ reduces $\mathrm{Nu}$ at the left wall and enhances at the right wall while $\mathrm{Nu}$ at the right wall enhances. Also $\mathrm{Nu}$ reduces with $\gamma>0$ and enhances with $\gamma<0$ at the left wall while Nu reduces at $\eta= \pm 0,1$.

An increase in $\mathrm{Sr}$ enhances the rate of mass transfer(Sh) at the left wall $(\eta=0)$ and reduces at the right wall $(\eta=+1)$.Higher the Hall parameter $(m)$ enhances $S h$ at the left wall and reduces at the right wall.Sh enhances at $\eta=0$ and reduces at $\eta=1$ with increase in rotation parameter( $R$ ). The rate of mass transfer reduces at $\eta=1$ and enhances at $\eta=0$ with increase in Nr. Higher the strength of the heat generating/absorbing heat sources smaller the rate of mass transfer at both the walls.An increase in chemical reaction parameter $(\gamma>0)$ reduces $S h$ at $\eta=0$ and enhances at $\eta=+1$ while a reversed effect is noticed in Sh with increase in $\gamma<0$.

Table2. Skin friction ( $\tau)$, Nusselt Number (Nu) and Sherwood Number (Sh) at $\eta=0 \& 1$

\begin{tabular}{|c|c|c|c|c|c|c|c|c|c|}
\hline \multicolumn{2}{|c|}{ Parameter } & $\tau_{\mathrm{x}}(\mathbf{0})$ & $\tau_{\mathrm{x}}(\mathbf{1})$ & $\tau_{\mathrm{z}}(\mathbf{0})$ & $\tau_{\mathrm{z}}(\mathbf{1})$ & $\mathbf{N u}(\mathbf{0})$ & $\mathbf{N u}(\mathbf{1})$ & Sh(0) & Sh(1) \\
\hline \multirow[t]{4}{*}{$\mathrm{m}$} & 0.5 & -6.4908 & 4.90622 & -0.20962 & 0.408574 & -0.999819 & -1.0001 & -0.638017 & -1.42464 \\
\hline & 1 & -6.40458 & 4.90374 & -0.237239 & 0.446312 & -0.999016 & -1.00052 & -0.638535 & -1.4245 \\
\hline & 1.5 & -6.37383 & 4.90245 & -0.224574 & 0.417362 & -0.99982 & -0.999985 & -0.637515 & -1.42529 \\
\hline & 2 & -6.37383 & 4.90245 & -0.224574 & 0.417362 & -0.999016 & -1.00052 & -0.638402 & -1.42463 \\
\hline \multirow[t]{4}{*}{$\mathrm{R}$} & 0.5 & -6.4908 & -6.4908 & -0.200962 & -0.209602 & -0.999819 & -1.00011 & -0.638017 & -1.42464 \\
\hline & 1 & -6.47338 & -6.47338 & -0.344238 & -0.344238 & -0.999017 & -1.00052 & -0.638861 & -1.42414 \\
\hline & 1.5 & -6.43735 & -6.43735 & -0.508451 & -0.508451 & -0.999821 & -0.999984 & -0.637873 & -1.42484 \\
\hline & 2 & -6.32977 & -6.32977 & -0.805523 & -0.805523 & -0.999016 & -1.00052 & -0.638458 & -1.42428 \\
\hline \multirow[t]{4}{*}{$\gamma$} & 0.5 & -6.4908 & 4.90622 & -0.20962 & 0.408574 & -0.999819 & -1.0001 & -0.638017 & -1.42464 \\
\hline & 1.5 & -6.48929 & 4.90811 & -0.209579 & 0.408616 & -0.999015 & -1.00052 & -0.526842 & -1.77103 \\
\hline & -0.5 & -6.49272 & 4.90386 & -0.209671 & 0.408521 & -0.999823 & -0.999982 & -0.78557 & -1.02414 \\
\hline & -1.5 & -6.49328 & 4.90318 & -0.209686 & 0.408505 & -0.999019 & -1.00052 & -0.830787 & -0.912426 \\
\hline \multirow[t]{4}{*}{$\mathrm{Sr}$} & 0.5 & -6.4908 & 4.90622 & -0.20962 & 0.408574 & -0.999819 & -1.0001 & -0.638017 & -1.42464 \\
\hline & 1 & -6.4908 & 4.90622 & -0.20962 & 0.408574 & -0.999017 & -1.00052 & -0.639457 & -1.4238 \\
\hline & 1.5 & -6.4908 & 4.90622 & -0.20962 & 0.408574 & -0.999821 & -0.999984 & -0.63823 & -1.42481 \\
\hline & 2 & -6.49081 & 4.90622 & -0.20962 & 0.408574 & -0.999017 & -1.00052 & -0.640561 & -1.42316 \\
\hline \multirow[t]{4}{*}{$\mathrm{Q}$} & 0.5 & -6.4908 & 4.90622 & -0.20 & 0.408574 & -0.999819 & -1.0001 & -0.638017 & -1.42464 \\
\hline & 1.5 & -6.4908 & 4.90622 & -0.20962 & 0.408574 & -0.999198 & -1.00016 & -0.638734 & -1.42457 \\
\hline & -0.5 & -6.4908 & 4.90622 & -0.20962 & 0.408574 & -0.99964 & -1.00035 & -0.638199 & -1.42434 \\
\hline & -1.5 & -6.49079 & 4.90623 & -0.20962 & 0.408574 & -0.998655 & -1.00125 & -0.63926 & -1.42323 \\
\hline \multirow[t]{4}{*}{$\beta$} & 0.2 & -6.4908 & 4.90622 & -0.20962 & 0.408574 & -0.999819 & -1.0001 & -0.638017 & -1.42464 \\
\hline & 0.4 & -5.8317 & 4.24151 & -0.1268 & 0.334284 & -0.998942 & -1.00068 & -0.582606 & -1.61266 \\
\hline & 0.6 & -5.21103 & 3.61529 & -0.0512105 & 0.266393 & -0.999675 & -1.00029 & -0.531981 & -1.79862 \\
\hline & 0.8 & -4.5973 & 2.99583 & 0.0213556 & 0.201137 & -0.998799 & -1.00097 & -0.486671 & -1.98853 \\
\hline \multirow[t]{4}{*}{$\mathrm{Nr}$} & 0.5 & -6.4908 & 4.90622 & -0.20962 & 0.408574 & -0.999819 & -1.0001 & -0.638017 & -1.42464 \\
\hline & 1.5 & -6.49079 & 4.90624 & -0.20962 & 0.408574 & -0.997411 & -1.00159 & -0.64068 & -1.4228 \\
\hline & 3.5 & -6.4908 & 4.90622 & -0.20962 & 0.408574 & -0.999821 & -0.999984 & -0.638023 & -1.42479 \\
\hline & 5 & -6.49076 & 4.90627 & -0.209619 & 0.408575 & -0.991815 & -1.00536 & -0.646847 & -1.41815 \\
\hline
\end{tabular}

\section{CONCLUSiOnS}

The effect of rotation, Hall effects, thermo-diffusion, squeezing, thermal radiation and heat source on convective heat and mass transfer of an electrically conducting fluid in a vertical channel has been analysed. It is found that an increase in $\mathrm{Sr}$, reduces $\mathrm{f}^{\prime} \& \mathrm{~g}$, while squeezing parameter $(\beta)$ enhances $\mathrm{f}^{\prime}$ and reduces g. The temperature and concentration enhance with Rotation and Soret parameter, reduces with squeezing parameter $(\beta)$. Higher the thermal radiation larger the temperature and smaller the concentration in the flow region. The Nusselt number enhances with $\mathrm{R}, \mathrm{Sr}, \beta$ and reduces with $\mathrm{Rd}$, while the Sherwood number reduces with $\mathrm{R}, \mathrm{Sr}$ and enhances with $\beta$ on $\eta=+1$. 


\section{REFERENCES}

[1] Adrian Postelnicu : Influence of magnetic field on heat and mass transfer by natural convection from vertical surfaces in porous media considering Soret and Dufour effects. Int. J. of Heat and Mass Transfer, V.47, pp.1467-1472 (2004).

[2] Agarwal, R.S and Dhanapal, C : Numerical solution to the flow of a micro polar fluid flow through porous walls of different permeability. pp. 325-336 (1987).

[3] Ahmed N and H.K. Sarmah : MHD Transient flow past an impulsively started infinite horizontal porous plate in a rotating system with hall current: Int J. of Appl. Math and Mech. 7(2) : 1-15, 2011.

[4] Alam, M.M and Sattar,M.A :Unsteady free convection and mass transfer flow in a rotating system with Hall currents, viscous dissipation and Joule heating ., Joural of Energy heat and mass transfer,V.22,pp.3139(2000)

[5] Alam, Md, Delower Hossain, M and Arif Hossain, M : Viscous dissipation and joule heating effects on steady MHD combined heat and mass transfer flow through a porous medium in a rotating system. Journal of Naval Architecture and Marine Engineering, V.2, pp.105-120 (2011).

[6] Aly, E.H. , Ebaid, A. and Elazem, N.Y.A. (2014):Analytical and numerical investigations for the flow and heat transfer of nanofluids over a stretching sheet with partial slip boundary condition, Applied Mathematics \& Information Science, Vol. 8 No. 4, pp. 1639-1645.

[7] Anwar Beg, O,Joaquin Zueco and Takhar,H.S:Unsteady magneto-hydrodynamic Hartmann-Couette flow and heat transfer in a Darcian channel with hall currents, ionslip, Viscous and Joule heating:Network Numerical solutions,Commun Nonlinear Sci Numer Simulat,V.14,pp.1082-1097(2009).

[8] Attia H.A., Ewis K.M. :Unsteady MHD Couette flow with heat transfer of a viscoelastic fluid under exponential decaying pressure gradient, Tamkang J. Sci. Eng. V.13 (4), pp.359-364(2010).

[9] Bachok, N., Ishak, A., Nazar, R. and Pop, I. (2010), "Flow and heat transfer at a general threedimensional stagnation point in a nanofluid", Physica B , Vol. 405 No. 24, pp. 4914-4918.

[10] Balasubramanyam $M$ : Effect of radiation on convective Heat and Mass transfer flow in a horizontal rotating channel communicated to Research India Publications, India (2010).

[11] Balla, C.S. and Kishan, N. (2014), "Finite element analysis of magnetohydrodynamic transient free convection flow of nanofluid over a vertical cone with thermal radiation", Proceedings of Instituation of Mechanical Engineers, Part N: Journal of Nanoengineering and Nanosystems. doi: 10.1740349914552879 .

[12] Barletta, A : Laminar mixed convection with viscous dissipation in a vertical channel. Int. J. Heat Mass Transfer, V.41, PP.3501-3513 (1998).

[13] Brewester, M.Q : Thermal radiative transfer and properties. John Wiley \& Sons. Inc. NewYork (1992).

[14] Buongiorno, J. (2006), "Convective transport in nanofluids", ASME Journal of Heat Transfer, Vol. 128 No. 3, pp. 240-250.

[15] Chamkha, A.J : MHD flow of a numerical of uniformly stretched vertical permeable surface in the presence of heat generation/absorption and a chemical reaction. Int. Comm. Heat Mass transfer, V.30, pp.413-422 (2003).

[16] Circar and Mukharjee : Effects of mass transfer and rotation on flow past a porous plate in a porous medium with variable suction in slip flow. Acta Cienica Indica, V.34M, No.2, pp.737-751 (2008).

[17] Das K. : Effects of heat and mass transfer on MHD free convection flow near a moving vertical plate of a radiating and chemically reacting fluid, J. Sib. Fed. Univ. Math. Phys. V.4, pp.18-31(2011).

[18] Domairry, G. and Aziz, A. (2009), “Approximate analysis of MHD squeeze flow between two parallel disks with suction or injection by homotopy perturbation method", Mathametical Problems in Engineering , Vol. 2009. doi: 10.1155/2009/603916.

[19] El.Mistikawy, T.M.A, Attia, H.A : The rotating disk flow in the presence of Strong magnetic field. Proc. $3^{\text {rd }}$ Int. Congr. of fluid mechanics. Cairo, Egypt. V.3, 2-4 January, pp 1211-1222 (1990).

[20] Ghouse, S.K : A note on steady and unsteady hydro magnetic flow in rotating channel in the presence of inclined magnetic field. Int. J. Eng. Sci., V.29, No.8, pp.1013-1016(1991).

[21] Gireesha B.J., Mahanthesh B. : Perturbation solution for radiating viscoelastic fluid flow and heat transfer with convective boundary condition in nonuniform channel with hall current and chemical reaction, ISRN Thermodyn., pp.1-14(2013).

[22] Greenspan,H.P:The theory of rotating fluids,Cambridge Univ.Press,UK(2013)

[23] Hamza, E.A. (1999), "Suction and injection effects on a similar flow between parallel plates",Journal of Physics D: Applied Physics, Vol. 32 No. 6, pp. 656-663. 
[24] Hamza, E.A. and Macdonald, D.A. (1981), “A fluid film squeezed between two parallel plane surfaces", Journal of Fluid Mechanics, Vol. 109, pp. 147-160.

[25] Hayat, T., Qayyum, A. and Alsaedi, A. (2015), “Three-dimensional mixed convection squeezing flow", Applied Mathematics and Mechanics - English Edition. , Vol. 36 No. 1, pp. 47-60.

[26] Hazem Ali Attia: Unsteady MHD flow near a rotating porous disk with uniform suction or injection. Fluid dynamics Research, V.23, pp.283-290.

[27] Indudhar Reddy, M, Narasimha Rao, P and Prasada Rao, D.R.V: Effect of chemical reaction and radiation absorption on unsteady MHD double diffusive convective flow of a viscous fluid past a semi-infinite porous plate. J. Pure \& Appl. Phys., V.23, No.2, pp.205-215 (2011).

[28] Jafarunnisa, S: Transient double diffusive flow of a viscous fluid with radiation effect in channels/Ducts, Ph.D Thesis, S.K.University, Anantapur, India (2011).

[29] Jayasudha J.S. and Siva Prasad R: "Soret and Dufour effect on convective heat and mass transfer flow past a vertical porous plate in a rotating fluid with chemical reaction, radiation absorption and dissipation", Ph.D. Thesis, Sri Krishnadevaraya University, Anantapuramu (2017)

[30] Kamalakar, P.V.S, Prasada Rao, D.R.V: Finite element analysis of chemical reaction effect on non-darcy convective heat \& mass transfer flow through a porous medium in vertical channel with heat sources. Int. j. Appl. Math \& Mech., V.13, pp.13-28(2012).

[31] 30a. Kalpana,K and Sreevani,M:Mixed convective squeezing three-dimensional hydromagnetic flow in a rotating channel with thermal radiation and thermo diffusion.,International Journal of Scientific and Innovative Mathematical research.,V.6(7),pp.28-42(2018)

[32] Krishna, D. V, Prasada Pao, D.R. V, Ramachandra Murty, A. S: Hydromagnetic convection flow through a porous medium in a rotating channel., J.Engg. Phy. and Thermo.Phy, V.75(2), pp.281-291.

[33] Madhavilatha,S and Prasada rao,D.R.V: Finite element analysis of convective heat and mass transfer flow past a vertical porous plate in a rotating fluid.,Int.Jour.Emerging and development,Vol.3,pp.202216,(2017)

[34] Madhusudhan Reddy, Y, Prasada Rao, D.R.V: Effect of thermo diffusion and chemical reaction on nondarcy convective heat \& mass transfer flow in a vertical channel with radiation. IJMA, V.4, pp.1-13 (2012).

[35] Mahanthesh B, Gorla R S R, Gireesha B J: "Mixed convection squeezing three-dimentional flow in a rotating channel filled with nanofluid", International Journal of Numerical Methods for Heat \& Fluid flow, Vol.26, Issue $5 \mathrm{pp}$

[36] Makinde O.D.: Free convection flow with thermal radiation and mass transfer past a moving vertical porous plate, Int. Commun. Heat Mass Transfer, V.32 pp.1411-1419(2005).

[37] Mohan, M: Combined effects of free and forced convection on magneto hydrodynamic flow in a rotating channel. Proc. Indian Acad. Sc., V.85, pp.383-401 (1977)

[38] Mohan, M, Srivatsava, K.K: Combined convection flows through a porous channel rotating with angular velocity. Proc. Indian Acad. Sci., V.87, p.14 (1978).

[39] Munawar, S., Mehmood, A. and Ali, A. (2012), "Three-dimensional squeezing flow in a rotating channel of lower stretching porous wall”, Computers and Mathematics with Applications, Vol. 64 No. 6, pp. 15751586.

[40] Mustafa, M., Hayat, T. and Obaidat, S. (2012), "On heat and mass transfer in the unsteady squeezing flow between parallel plates”, Meccanica, Vol. 47 No. 7, pp. 1581-1589.

[41] Muthcuumaraswamy R., Senthil G.K.: Heat and mass transfer effects on moving vertical plate in the presence of thermal radiation, Theor. Appl. Mach. Pp. 35-46(2014).

[42] Muthcuumaraswamy, R, Dhanasekhar, N and Eswar Prasad, G: Rotation effects on flow past an accelerated isothermal vertical plate with chemical reaction of first order. IJMA, V.3, No.5, pp.2122-2129 (2012).

[43] Nanda R.S and Mohanty, H.K: Hydromagnetic flow in a rotating channel. Appl. Sci. Res., V.24, pp.6578(1970).

[44] Pal D., Talukdar B.: Perturbation analysis of unsteady magnetohydrodynamic convective heat and mass transfer in a boundary layer slip flow past a vertical permeable plate with thermal radiation and chemical reaction, Commun. Nonlinear Sci. Numer. Simul. V.15 (7), pp.1813-1830(2010).

[45] Prasada Rao, D.R.V, Krishna, D.V and Debnath, L: Combined effect of free and forced convection on MHD flow in a rotating porous channel. Int. J. Math and Math. Sci., V.5, pp.165-182 (1982).

[46] Rajasekhar, N.S, Prasad, P.M.V and Prasada Rao, D.R.V: Effect of Hall current, Thermal radiation and thermo diffusion on convective heat and mass transfer flow of a viscous, rotating fluid past a vertical porous plate embedded in a porous medium. Advances in Applied Science Research, V.3, No.6, pp.34383447 (2012). 
[47] Rashidi, M.M., Shahmohamadi, H. and Dinarvand, S. (2008), “Analytic approximate solutions for unsteady two-dimensional and axisymmetric squeezing flows between parallel plates",Mathematical Problems in Engineering, Vol. 2008, 12pp.

[48] Sarojamma, G and Krishna, D.V: Transient Hydromagnetic convection flow in a rotating channel with porous boundaries. Acta Mechanica, V. 39, p.277 (1981).

[49] Sarpkaya T: Flow of non-Newtonian fluids in a magnetic field, AIChE J. V.7 pp.324-328(1961).

[50] Seth G.S. and Ghosh, S.K: Ind J. Eng. Sci., V.24, No.7, pp.1183-1193 (1986).

[51] Seth G.S., Ansari Md.S., Nandkeolyar R.: MHD natural convection flow with radiative heat transfer past an impulsively moving plate with ramped wall temperature, Heat Mass Transfer V.47, pp. 551-561(2011).

[52] Seth,G.S,Ansari,S and Ahmad,N : Acta Ciencia Indica,V.34M, No.4, p.1849 (2008)

[53] Sheikholeslami, M., Hatami, M. and Ganji, D.D. (2014), "Nanofluid flow and heat transfer in a rotating system in the presence of a magnetic field", Journal of Molecular Liquids, Vol. 190, Februrary, pp. 112120.

[54] Singh, K.D and Mathew: An oscillatory free convective MHD flow in a rotating vertical porous channel with heat sources. Ganita, V.60, No.1, pp.91-110 (2009).

[55] Sivaiaih, S: Thermo diffusion effects on convective heat and mass transfer through a porous medium in ducts. Ph.D Thesis, SKU, Anantapur, India (2004).

[56] Soundalgekar, V.M and Bhat, J.P: MHD flow and heat transfer in viscous electrically conduction fluid in a rotating channel with conducts plates. Applied Energy, V.20, pp.141-151 (1985).

[57] Sreerangavani,K,Rajeswara Rao,U and Prasada Rao,D.R.V:Effect of thermo-diffusion on Mhd convection heat and mass transfer flow of chemically reacting fluid through a porous medium in a rotating system.,Presented in $2^{\text {nd }}$ International conference on Applicatrions of Fluid dynamics,July,(2014)

[58] Sreevani,M : Mixed convective heat and mass transfer through a porous medium in channels with dissipative effects, Ph.D thesis, S.K.University, Anantapur, India (2002).

[59] 57a. Sreevani,M: Hydromagnetic convection squeezing three-dimensional flow in a rotating channel., Int.J.Sci.Mathematical research,V.6(7),pp.1-15(2018)

[60] Stefan, M.J. (1874), "Versuch U ber die scheinbare adhesion Sitzungsberichte der Akademie der Wissenschaften in Wien", Mathematik-Naturwissen, Vol. 69, pp. 713-721.

[61] Sukanya J.S and Leelarathnam A: "combined influence of Hall Currents and Soret effect on convective heat and mass transfer flow past vertical porous stretching plate in rotating fluid and dissipation with constant heat and mass flux and partial slip", IJATED (2018)

[62] Turkyilmazoglu M., Pop I.: Soret and heat source effects on the unsteady radiative MHD free convection flow from an impulsively started infinite vertical plate, Int. J. Heat Mass Transfer, V. 55, pp. 76357644(2012).

[63] Uwanta I.J., Omokhuale E.: Viscoelastic fluid flow in a fixed plane with heat and mass transfer, Res. J. Math. Stat. V.4 (3), pp.63-69(2012).

[64] Zanchini, E: Effect of viscous dissipation on mixed convection in a vertical channel with boundary conditions of the third kind. Int. J. of Heat and Mass Transfer, V.41, pp.3949-3959 (1998). 


\section{AUTHORS' BIOGRAPHY}



Smt. Suguna H.G, working as Assistant professor, at department of Mathematics, Govt college for Boys, Chintamani, Chikkaballapurdist, Karnataka. She has completed her M.Sc., in the year 2005 and M.Phil in the year 2007 from Alagappa university, Karaikudi, Tamilnadu. Presently perusing PhD at Dravidian University, Kuppam, Andrapradesh. Her area of research is fluid mechanics. She is having a teaching experience of 22 years as a teacher and Assistant professor. She has presented 4 papers at national and international conferences and seminars. She was the organizing committee member for the Rastriyakannadavignanasammelana held at Bangalore north University in the year 2018. BOE member of Mathematics during 2018-19 for Bangalore north University and BOS member for VHD Home science college, Bangalore. Active member for Bangalore University Mathematics Forum.

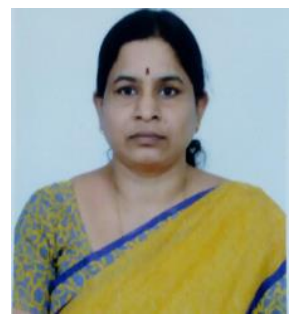

Dr. Bhuvanavijaya. R, presently working as professor and Head, at department of mathematics, JNTUA college of Engineering, Anantapuram, Andhra Pradesh. She has completed her M.Sc., in the year 1990 from SK university Anantapur, M.Phil in the year 1998 from SK university and Doctoral degree in the year 2000 and her area of research is linear algebra and fluid mechanics. She is having a teaching experience of 28 years as a lecturer, an Adhoc lecturer, as an assistant professor and presently working as professor at JNTUCEA. She has published 55 articles in reputed national and international journals. She has presented 12 papers at national and international conferences and seminars. She has organized National workshop on mathematical and computational methods in fluid dynamics in the year 2013. In her academic career 8 Research scholars had been awarded doctoral degree under her guidance and also presently guiding 5 research scholars.

Citation: Suguna H.G \& Bhuvana Vijaya R (2019). Effect of Hall Current on Mixed Convection Squeezing Three-Dimensional Flow in a Rotating Channel with Thermal Radiation and Heat Sources. International Journal of Scientific and Innovative Mathematical Research (IJSIMR), 7(3), pp.40-52. http://dx.doi. org/10.20431/2347-3142.0703005

Copyright: (1) 2019 Authors, This is an open-access article distributed under the terms of the Creative Commons Attribution License, which permits unrestricted use, distribution, and reproduction in any medium, provided the original author and source are credited. 